\title{
Zwei Bemerkungen zum Papyrus Insinger
}

Die Weisheitslehre des Papyrus Insinger ist ein Text, der nach dieser Handschrift in vielen Punkten weiterhin Schwierigkeiten bereitet und immer noch nicht als völlig verstanden gelten darf. In der Nachfolge A. Voltens, der sich mit Fragmenten von Parallelen in Kopenhagen beschäftigte, sieht sich die Handschrift zahlreichen verbessernden Eingriffen von demotistischer Seite ausgesetzt. Aufgrund der Kopenhagener Abweichungen emendierte Volten einige Lesarten des pInsinger in stillschweigender und impliziter Ablehnung des Prinzips der lectio difficilior, da die Kopenhagener Handschriften leichter verständliche und daher vermeintlich korrektere Lesarten überliefern, und ließ meist die für heutige Leser zugänglicheren Varianten der Carlsberg-Parallelen als die richtigeren gelten ${ }^{1}$. Nun ist es unbestritten, daß bei einem derart langen Text dem demotischen Schreiber Fehler unterlaufen sein mögen. Es ist jedoch zu fragen, ob nicht eine größere Bescheidenheit dem Ägypter gegenüber angezeigt wäre, denn jede Emendierung impliziert die Annahme, daß der Emendierende besser Demotisch könne als der Ägypter, der pInsinger in seiner Muttersprache geschrieben hat.

Eine jüngste Diskussion einer Stelle des pInsinger (XVIII 5-12) ist von J. F. Quack veröffentlicht worden, mit der ich mich in Teilaspekten bereits in SAK 29 kritisch auseinandergesetzt habe. Aufgrund zweifelhafter Emendierungen in das demotische Original gelangte er zu der Deutung, daß sich die Stelle auf die am Grab tatsächlich ausgeführten Totenriten inklusive

A. Volten, Kopenhagener Texte zum demotischen Weisheitsbuch (Pap. Carlsberg II, III Verso, IV Verso und V). Analecta Aegyptiaca 1 (Kopenhagen 1940). Id., Das demotische Weisheitsbuch, Studien und Bearbeitung. Analecta Aegyptiaca 2 (Kopenhagen 1941). einer angeblichen Bootsfahrt bezöge ${ }^{2}$. Seine Korrektur von $w r$ iyh (genauer wohl tatsächlich $w r$ iyh $<. w>\mathrm{zu}$ transliterieren) „Oberdämon, Oberster der Ach-Geister" zu wr iry „Balsamierer" ist aufgrund paläographischer Erwägungen sicher abzulehnen, den Übersetzungsvorschlag „Bootsfahrt" statt „douceur“ bzw. „inflammation" für hne hatte ich aber zwischenzeitlich, wenngleich zögernd und zweifelnd, akzeptiert. Seine Übersetzung ist dennoch durch diese Eingriffe nicht unmittelbar verständlicher geworden und muß erst mit beträchtlichem Aufwand erklärt werden. Dagegen hatte ich die Auffassung vertreten, die fragliche Passage befasse sich nicht mit dem diesseitigen, sondern vielmehr dem jenseitigen Schicksal des Verstorbenen. Eine Weisheitslehre habe nicht nur das Diesseits, sondern auch das Leben nach dem Tode im Blick, wofür auch spräche, daß es guten Grund gäbe anzunehmen, die Lehre des Anchscheschonqi sei einem Verstorbenen mit ins Grab gegeben worden. Sowohl das Problem der Übersetzung von $h n e^{3}$ in pInsinger XVIII 12 und davon ausgehend die Interpretation von XVIII 5-12 als auch das Problem einer Weisheitslehre im funerären Kontext möchte ich im vorliegenden Aufsatz behandeln. Es wird sich zeigen, daß beide Probleme miteinander innerlich verbunden sind.

${ }^{2}$ J. F. Quack, „Balsamierung und Totengericht im Papyrus Insinger", in: Enchoria 25 (1999) 30-38. M. A. Stadler, „War eine dramatische Aufführung des Totengerichts Teil der ägyptischen Totenriten?", in: SAK 29 (2001) 331-348.

${ }^{3}$ Ich habe Dr. Mark Smith, University College Oxford, dafür herzlich und vielmals zu danken, mir seine Ideen zu hne mitgeteilt und mir gestattet zu haben, sie hier heranziehen zu dürfen. Für weitere Verbesserungsvorschläge bin ich Prof. Karl-Theodor Zauzich zu Dank verpflichtet. Ferner hat Frau Dr. Sandra Lippert eine vorläufige Fassung kritisch durchgesehen. 


\section{Papyrus Insinger als Grabbeigabe}

Zunächst zur Diskussion des funerären Kontextes. Zwar konnte für die Lehre des pBM 10508 (Anchscheschonqi) eine enge Verbindung mit dem Totentexte überliefernden demotischen pBM 10507 überzeugend, jedoch nur mittelbar nachgewiesen werden ${ }^{4}$. Ein expliziter Hinweis aber, der nichts an Deutlichkeit zu wünschen übrig läßt, auf pInsinger im Grabkontext ist der Schluß des Papyrus selbst:

p3 mnk $t i^{5}$ rh 'me rnpy by=f $\varsigma^{\varsigma}$ ' nhh d. d.t

$p 3-h b s 3 \underline{d}-h r-p 3-{ }^{-c} n r$ by $=f r$ sms w wir-skr

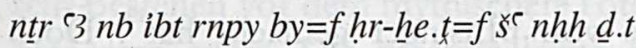

„Das Ende der Lehre. Sein Ba möge sich verjüngen für immer und ewig,

des $\mathrm{Pa}-\mathrm{Heb}$, Sohn des Dje-her-pa-a'an, indem seine Seele Osiris-Sokar dienen wird,

dem großen Gott, dem Herren von Abydos. Seine Seele verjünge sich auf $\mathrm{ihm}^{6}$ für immer und ewig." (XXXV 13-15).

Dieser Schluß steht am Ende einer bemerkenswerten Folge von Sentenzen, die ebenso ein bezeichnendes Licht auf die Observanz der Lehre des pInsinger wirft. Die letzte, diesem Kolophon unmittelbar voranstehende 25. Lehre „Der Weg, dich vor Vergeltung in acht zu nehmen, damit dich nicht ein Teil davon treffe." nimmt eine etwas überraschende Wendung: In XXXV 1 wird noch ein Satz über Gewalt, Not, Schmähung und Unbarmherzigkeit, die nie zur Ruhe kommen, gegeben, worauf in den Zeilen 2 und 3 zwei Sätze folgen, die an die erste Folge des negativen Sündenbekenntnisses in der ein-

M. Smith, „Budge in Akhmim“, in: C. Eyre/ A. Leahy/L. Montagno Leahy (Hgg.), The Unbroken Reed. Studies in the Culture and Heritage of Ancient Egypt in Honour of A. F. Shore. EES Occasional Publications 11 (1994) 293-303.

Zweifel an $t i$ wurden von K.-Th. Zauzich, LÄ IV, Sp. 898, geäußert.

Siehe W. Erichsen, Demotisches Glossar (Kopenhagen 1954) $321 \mathrm{zu}$ hr-he.t. H.J. Thissen, in: Weisheitstexte II. Texte aus der Umwelt des Alten Testaments III, 2 (Gütersloh 1991) 319, übersetzt mit $h r$,und“" , „... seine Seele und sein Leib“. Der Ba auf dem Toten ist jedoch ein in Ägypten gängiges Bild.

leitenden Rede des 125. Totenbuchkapitels erinnern:

(2) $b n-p w=y m w h$ ir

$m t . t b n<. t>h r(?)^{7}$

h3.t=y $p 3$ ntr $i r-r h=f$

(3) $b n-p w=y$ qby $r k y$

bn-pw ky th3 $n$ rn $=y$
(2) Ich habe nicht ge brannt, eine böse Sache zu tun auf (?) meinem Herzen Gott weiß es.

(3) Ich habe nie Zwang ausgeübt gegen einen anderen. Ein anderer hat nie geschädigt in meinem Namen.

Die Nähe zum negativen Sündenbekenntnis des 125. Totenbuchkapitels ist augenfällig, wobei die drei mit $b n-p w$ konstruierten Sätze des pInsinger so allgemein sind, $\mathrm{da} ß$ sie fast als eine Zusammenfassung des negativen Sündenbekenntnisses gelten können. Die Nähe wird noch deutlicher, wenn die Sätze des pInsinger der demotischen Übersetzung von $\mathrm{Tb} 125$ gegenübergestellt werden, wie sie in pBN 149 überliefert ist ${ }^{8}$. Drei Beispielsätze aus dem sogenannten demotischen Totenbuch mögen genügen:

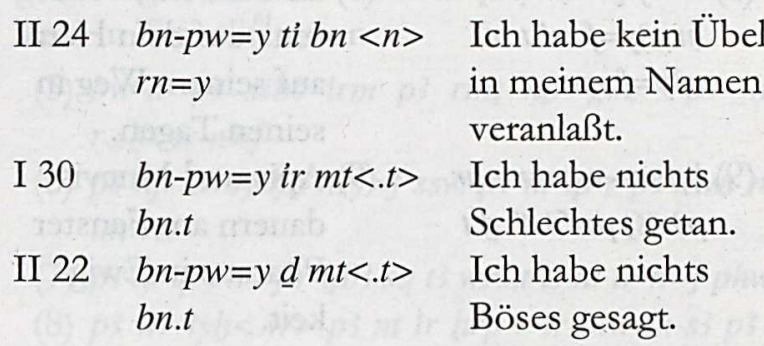

Bis hin zu H.J. Thissen, in: Weisheitstexte II. Texte aus der Umwelt des Alten Testaments III, 2 (Gütersloh 1991) 319, wird hier ein Fragezeichen gesetzt, ohne daß das Zeichen gelesen wird. Es ist einigermaßen verkleckst und daher schwer zu erkennen. Grammatikalisch fehlt nichts ohne das Wort, wenn man mit Thissen übersetzt: „Ich habe nie darauf gebrannt, Böses zu tun ...? ...; Gott kennt mein Herz." Eine befriedigende Lösung stellt mein Vorschlag allerdings auch nicht dar.

Editio princeps von F. Lexa, Das demotische Totenbuch der Pariser Nationalbibliothek (Papyrus des Pa-Month). Demotische Studien 4 (Leipzig 1910), Neuedition von M.A. Stadler, Der Totenpapyrus des Pa-Month (P. Bibl. nat. 149), Studien zum Altägyptischen Totenbuch 6 (Wiesbaden 2003). 
Im pInsinger XXXV schließt sich wohl eine Bitte um Vergebung an, um dann nochmals das Gottvertrauen zu betonen:

(4) $p 3$ lwh i.ir=y iw $b n-p w=y r h=f$ $t w=y \operatorname{sbh}[\ldots]$

(5) $\ulcorner\longleftarrow=y n p 3 n t r$ $h t p=f-n=y$ ir $=f-n=y$ hny iw mn [...]

(6) $i w=f \lg r w s n t 3$ $w \underline{d}$.t iw $\mathrm{mn} w \underline{d} 3$

(7) $i w=f t i c h i w ~ m n$ hm-ir.t qsi3.t $<$ nfr.t>

(8) $i w=f h 3^{e} h r h 3 . t=k$ $h r t 3 y=f$ mi.t $n$ $n 3 y=f s s w$

(9) hp mr-wr mne hr

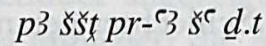

(10) $i w=w$ ir $m t . t$ $\{n\}-\} n f r . t n p 3 n t$ $i w=f s d m-n=y$ irm. p3 $n t i w=f \underline{d}[\ldots]$

(11) ḩ.t rmt $r h$ h $p 3 y=f$ db3 ir.t $p^{3}$ ntr $r-h r=y$
(4) Das Vergehen, welches ich begangen habe, indem ich es nicht wußte, ich bitte [um Vergebung... .]

(5) Ich rief zu Gott, so $\mathrm{daß}$ er mir gnädig sei, er mir ein hny ${ }^{9}$ sei, indem es nicht gibt [...]

(6) Er wird beseitigen die Sorge um Wohlbefinden, indem es keinen Rest gibt.

(7) Er wird eine Lebenszeit geben, indem es kein Unglück gibt, und ein $<$ schönes $>$ Begräbnis.

(8) Er wird sich verlas$\operatorname{sen}^{10}$ auf dein Herz auf seinem Weg in seinen Tagen.

(9) Apis und Mnevis dauern am Fenster Pharaos in Ewigkeit.

(10) Sie werden ein gutes Werk tun an dem, der auf mich hört, und dem, der sagen wird [...]

(11) Das Herz eines Weisen ist seine Vergeltung, das Auge Gottes ist auf ihm ${ }^{11}$.
9 Zur Übersetzung vgl. die Ausführungen unten.

${ }^{10}$ This sen, in: TUAT III 2, 319 übersetzt hier präsentisch, zuvor und danach aber wieder futurisch.

${ }^{11}$ „Das Herz eines Weisen - seine Vergeltung ist das Auge Gottes ........." (Thissen, in: TUAT III 2, 319.)
(12) $h 3 . t \mathrm{~s} s b_{3} \mathrm{iw}=\mathrm{ftm}$ $r h n h 3 . t[\ldots]$

(12) Das Herz eines Gottlosen, er kennt nicht ein Herz [. . . ]

Der Nachsatz des pInsinger ist in der einschlägigen Insinger-Literatur bislang nicht ausreichend gewürdigt und von Quack in seinen Überlegungen gar nicht beachtet worden ${ }^{12}$, bekommt aber angesichts der jüngst geführten Diskussion ein neues Gewicht, nachdem dadurch der Text zu einer Art Totentext, mindestens aber einer Grabbeigabe wird. Spiegelberg ist, soweit ich das sehen kann, der einzige, der sich überhaupt Gedanken über die Deutung des Schlusses von pInsinger gemacht hat ${ }^{13}$. Doch trifft seine Interpretation des pInsinger als Totenbeigabe für eine Ibismumie wohl nicht das Richtige. Der Ibis sei - so Spiegelberg - das heilige Tier des Thot, der seine Freude an dem Weisheitstext finden möge, um dann dem verstorbenen Ibis die Seligkeit zu verschaffen. Spiegelberg hat sich wohl von dem Eigennamen $p 3-$ $h b$ wörtl. „der Ibis“ in die Irre führen lassen, was er dann mit bei Ibis-Mumien gefundenen Papyri in Verbindung brachte. Allerdings ist es unwahrscheinlich, einem Ibis noch eine Filiation beizugeben, wie das hier geschehen ist. Der funeräre Kontext der Lehre des Anchscheschonqi, den M. Smith nachweisen konnte, zeigt, daß Weisheitstexte als Grabbeigabe von Menschen durchaus belegt sind, was im Lichte des Schlußsatzes auch für den pInsinger anzusetzen sein wird ${ }^{14}$.

${ }^{12}$ Bei M. Lichtheim, Late Egyptian Wisdom Literature in the International Context. A Study of Demotic Instructions (Freiburg Schweiz/Göttingen 1983), gar nicht behandelt. In der Übersetzung von H. -J. Thissen, in: TUAT III 2, 280 f. und 319, nicht weiter kommentiert.

${ }^{13}$ W. Spiegelberg, „Die Schlussworte des demotischen Papyrus Insinger", in: OLZ 3, 1900, 268f. Der Schlußsatz des pInsinger wird lediglich mit der Schlußfolgerung, pInsinger käme demnach aus einem Grab, von M. Smith und H.-J. Thissen kurz erwähnt: M. Smith, „Aspects of Indigenous Religious Traditions", in: A. Egberts/B. P. Muhs/J. van der Vliet (Hgg.), Perspectives on Panopolis. An Egyptian Town from Alexander the Great to the Arab Conquest. Papyrologica Lugduno-Batava 31 (Leiden/Boston/Köln 2002) 237. H.-J. Thissen, ,Achmim und die demotische Literatur", in: Perspectives on Panopolis $260 \mathrm{mit}$ Anm. 51.

${ }^{14}$ M. Smith, Fs Shore 293-303. 
Die Relevanz einer Weisheitslehre für den Verstorbenen und sein Eingehen in das Jenseits speist sich aus verschiedenen Quellen. Einmal wies sich der Besitzer damit vor Thot als Weiser und treuer Gefolgsmann aus und bewies seine fromme Lesetätigkeit ${ }^{15}$. Das ist möglich, aber es sind auch noch zwei weitere Deutungen möglich, wobei alle Interpretationen sich nicht gegenseitig ausschließen.

Einmal soll der Text vermutlich ebenso belegen, daß sich der Verstorbene, der den Text auf seine Reise in den Westen mitnimmt, zu Lebzeiten um ethisches Verhalten bemühte, was sein Bestehen vor dem mythischen Totengericht befördern sollte. Die gesamte letzte Kolumne hat einen transzendenten Charakter und versteckt ihren Blick auf das Jenseits nicht. Der Zusammenhang mit Tod und Jenseits ist für ägyptische Lebenslehren nicht neu. Vielmehr unterwies schon früher als literarischer Topos ägyptischer Lehren der alternde und somit dem Tod nahe Vater seinen Sohn, und die Ethik hatte ihren festen Platz in den Gräbern, in denen die Autobiographie das Leben entsprechend ihren Idealen für die Nachwelt festhielt ${ }^{16}$.

Der Schlußsatz wäre ebenso ein Beleg für die Pietät des Sohnes, der den Text niederschrieb, so wie $z$. B. auch Menkara $\mathrm{pBN} 149$ für seinen Vater Pa-Month schrieb ${ }^{17}$. Der Sohn gab dem Vater pInsinger zum zweifachen Beleg ins Grab mit: Einmal wird damit die ethische Unterweisung durch den Vater bezeugt und so implizit dessen Befolgung der ethischen Prinzipien, zum anderen zeigt der Sohn den Erfolg der Unterweisung und beteuert, sich daran zu halten und für die totenkultische Versorgung des Vaters zu sorgen. In dieser Sicht würde pInsinger in sich das Beziehungsgeflecht zwischen totem Vater

${ }^{15}$ Zur Beschäftigung mit Texten als frommer Tat vgl. Quellen wie: P. Oxy. XI.1381, bes. 32-52, 191-198. Beschäftigung mit Texten als typische Priestertätigkeit steht in satirischer Überzeichnung und/ oder als Faust-Motiv im Zentrum des ersten SetneRomans. Vgl. auch: A. v. Lieven, „Wissen, was die Welt im Innersten zusammenhält oder Faust in Ägypten?", in: Journal of Ancient Near Eastern Religions 2 (2002) 75-89.

J. Assmann, Tod und Jenseits im Alten Ägypten (München 2001) 73-78.

pBN 149 III 27-30. und lebendem Sohn, zwischen Jenseits und Diesseits verkörpern und so einmal mehr fest in der Tradition der ägyptischen Lebenslehren verwurzelt erscheinen. Die Aussage der zwar stark beschädigten ersten publizierten Kolumne, die freilich nicht die erste Kolumne der Handschrift war und insofern nicht das Generalthema der Lehre enthielt ${ }^{18}$, ließe sich in diese Richtung rekonstruieren, indem dort die Sohnespietät und deren vorteilhafte Ergebnisse für den Sohn ausgeführt wurden ${ }^{19}$. Die Bedeutung des Weisheitstextes des pInsinger für das Jenseits ist somit verständlich, und die jenseitige Perspektive der Lehre, wie sie Quack bei seiner Deutung von pInsinger XVIII 5-12 ablehnt, gewinnt dadurch an zusätzlicher Unterstützung.

\section{Nochmals zur vermeintlichen Seefahrt (hne) in pInsinger XVIII 10: Anubis als Oberdämon, Freund und Richter}

Die Diskussion über hne und die letztliche Deutung von pInsinger XVIII 5-12 muß vom Text und seinem Kontext innerhalb des pInsinger ausgehen. Hier zunächst der demotische Text in Transliteration:

(5) bw-ir p3 s3b3 irm p3 rmt ntr gm ${ }^{c} p^{3} c^{c}$ $r . s \underline{h}=w-n=f$

(6) p3 nfr ir šy hnn n3y=f ssw p3 nt ip $r$ p3 mwt n$i m=w$

(7) $p 3$ nt ip $r-r=f r-d b 3$ sq $t 3$ wr $3 . t$ t3 nt ir-n=f phw

(8) $p 3$ wr iyh $\underline{h}<. w>p 3$ nt ir hrp $r$ ir btw $m-s 3$ p3 $\underline{t}$. p $3 \underline{t} 3 w$

(9) syf sntr h hsmn hmm3 phrr.t hm.t phr.t n3y=f shy.w

(10) hne iw bw-ir=f $n^{\ulcorner} p 3$ nt mwhmh $n n 3 y=f i w f . w$

(11) $b w$-ir=f rh $\underline{d} h h^{\top} \underline{d} r . t=k$ hn $p^{3}$ btw $n$ p3 i.ir shyh

(12) $t 3 \underline{h^{c}} . t p^{3}$ rmt nt $\underline{t}$ tms $=f h r$ p 3 tw irm $t 3 y=f$ qse.t

Die vorläufige Übersetzung:

(5) Der Frevler und der fromme Mann finden nicht die Länge der Lebenszeit, die ihnen zugeschrieben ist.

(6) Der Gute, in dessen Tagen Glück war, ist der, der währenddessen an den Tod denkt.

${ }^{18} \mathrm{Vgl}$. K.-Th. Zauzich nach Lichtheim, Late Egyptian Wisdom Literature 107-109.

Vgl. auch pInsinger II $14 \mathrm{f}$. 
(7) Der, der daran denkt wegen des Sparens, die Unheilsdämonin ist die, die ihm ein Ende schafft.

(8) Der Oberste der Ach-Geist $<$ er $>$ ist der, der früh kommt, um Leiden zu machen nach dem Nehmen des Atems.

(9) Zedernöl, Weihrauch, Natron, Salz und "heiße Medizin“ sind Medizin für seine Wunden.

(10) Ein hne, indem er nicht gnädig ist, ist der, der sein Fleisch *malträtiert.

(11) Er kann nicht sagen „Tue deine Hände weg“" bei der Tortur dessen, der *examiniert.

(12) Das Ende eines frommen Menschen: Er wird begraben auf dem Berg zusammen mit seiner Grabausstattung.

Quack übersetzte ${ }^{20}$ :

(5) Frevler und Mann Gottes kennen nicht die Art der Lebenszeit, die ihnen zugeschrieben wurde.

(6) Wer vom Schicksal in seinen Tagen begünstigt wurde, ist es, der in ihnen an den Tod denkt.

(7) Wer nur zum Sparen an ihn denkt, dem setzt die Unheilsdämonin ein Ende.

(8) Der $w r$-iyh $<w r$-irin $>$ ist es, der ihm zuerst Strafe zufügt, nachdem man ihm den Lebensatem genommen hat.

(9) Pech, Weihrauch, Natron, Salz und das „heiße Medikament“ sind Heilmittel für seine Wunden.

(10) Eine unbarmherzige Seefahrt ist es, die seinen Körper malträtiert.

(11) Er kann nicht „Halt ein“ sagen bei der Bestrafung durch den, der examiniert hat.

(12) Das Ende des Mannes Gottes ist, ihn mit seiner Grabausstattung in der Nekropole zu begraben.

Zum Teil schließe ich mich den Quackschen Verbesserungsvorschlägen an, die ich hier nicht weiter kommentieren muß, da dies schon von ihm erledigt wurde (so die Unheilsdämonin $t 3$ wr3.t, das absichtlich und zurecht mit Vorsicht allgemein gehaltene $m w h m h$, "malträtieren" und

\footnotetext{
${ }^{20}$ Enchoria 25 (1999) 28.
}

shyh „untersuchen, examinieren “) $)^{21}$. Den wr-iry für einen an der Einbalsamierung Beteiligten (den, der die Eingeweide entfernt), den Quack statt des $w r$-iyh „Oberdämonen, Obersten der Ach-Geister" gerne gesehen hätte, habe ich an anderer Stelle mit ausführlicher Diskussion abgelehnt $^{22}$. Daran habe ich damals vorläufige Erwägungen zur Deutung der Stelle angeschlossen, die sich aber vor allem mit der $\mathrm{m}$. E. fälschlichen Annahme einer szenischen Aufführung des Totengerichtes am Grab auseinandersetzten.

Hier soll nun jedoch aus Anlaß des Vorschlages einer Neuübersetzung von hne in XVIII 10 der Versuch einer von Quack noch deutlicher abweichenden Gesamtinterpretation der Stelle unternommen werden. Die Argumentation ist zweigeteilt: Zuerst will ich versuchen, sowohl paläographisch als auch lexikographisch \&ul in neuer Übersetzung $z$ begründen, wobei ich mich aber nicht mit einem Verweis auf die Diskussion des Wortes und seiner Lesung und Übersetzung vor Quack beschränken kann, sondern sie teilweise neu aufrollen muß. Ich schließe daran dann die inhaltliche Untersuchung an, die klären will, ob sich die Übersetzung aufgrund ihres Sinnes durch andere, insbesondere totenreligiöse Texte erhärten läßt.

Hier ein Überblick über die bisher vor der oben zitierten Quackschen gegebenen Übersetzungen:

„La douceur (bne) qui enflammerait son corps, n'arrive pas. ${ }^{\text {(23 }}$

"An inflammation that has no mercy burns his body. ${ }^{624}$

${ }^{21}$ Allerdings weiche ich in der Auffassung der periphrastischen Form von i.ir ab und sehe es als Periphrase des Aorist. Vgl. M. Lich theim, „On the Participle iir in Demotic", in: D. W. Young (Hg.), Studies presented to H. J. Polotsky (East Gloucester, Massachusetts 1981) 463-471. Jetzt außerdem: M. Depauw, Two Notes on the Demotic Participle or how i.ir Became Theologically Relevant, in: LingAeg 10 (2002), $101-121$.

${ }^{22}$ SAK 29, $340 \mathrm{ff}$.

${ }^{23}$ F. Lexa, Papyrus Insinger. Les enseignements moraux d'un scribe égyptien du premier siècle après J.-C. I (Paris 1926) $57 \mathrm{f}$.

${ }^{24}$ M. Lichtheim, Ancient Egyptian Literature III (Berkeley/Los Angeles/London) 215 Anm. 59. Ead., Late Egyptian Wisdom Literature 215. 
„Unbarmherzige Entzündung verbrennt sein Fleisch. ${ }^{625}$

Lichtheim zieht für ihre Übersetzung eine Etymologie von älterem $h n$ „krankhafter $\mathrm{Zu}$ stand der Haut in Folge grosser Hitze" und hnn „entzündet sein o. ä.“ in Betracht" ${ }^{26}$, was dann von Thissen übernommen wurde.
Zunächst sollen die Wörter untersucht werden, die im pInsinger hne oder hnyy ge-schrieben werden, um so eine Grundlage für die Diskussion zur Übersetzung des fraglichen Wortes zu erhalten. Diese Wörter werden zunächst allein nach ihrem graphischen Bild sortiert, wobei die Stellenangaben nur die Herkunft des spezifischen Facsimiles angeben. Die Übersetzungen geben den Kontext dieser konkreten Stelle.

\begin{tabular}{|c|c|c|}
\hline (1) & X 18 fisulus & $\begin{array}{l}\text { hnt „Dummheit" („Sage ihm nichts Böses im Tadeln deiner } \\
\text { Dummheit.“) }\end{array}$ \\
\hline (2) & & $\begin{array}{l}\text { hne „Nähe“ („Von Herzen sollst du ihm dienen (in) seiner Nähe } \\
\text { wie (in) seiner Ferne. }{ }^{27}\end{array}$ \\
\hline (3) & XII 12 \& (u, & $\begin{array}{l}\text { hne „Tölpel, Dummkopf, Störenfried“ („Vertraue nicht einem } \\
\left.\text { Tölpel zu irgendeiner Zeit in einer Angelegenheit. }{ }^{\circ}\right)^{28}\end{array}$ \\
\hline (4) & IV 5 f cosol & $\begin{array}{l}\text { hne „störend“ („Eine störende Zunge eines dummen Menschen } \\
\text { ist sein Schwert des Abschneidens seiner Lebenszeit.“) }\end{array}$ \\
\hline (5) & XIII 17 닐 & $\begin{array}{l}\text { hne „sich nähern“ („Der dumme Mensch ist der, der Streit ent- } \\
\text { stehen läßt, und der, der sich ihm nähert, indem er fällt." }{ }^{29}\end{array}$ \\
\hline (6) & XXVI 12: ir ents & $\begin{array}{l}\text { hne „Umgang (pflegen)“ („Pflege keinen Umgang mit einem } \\
\text { anderen, wenn } \mathrm{Haß} \text { in seinem Herzen ist.") }\end{array}$ \\
\hline (7) & XVIII 10 kiles & $\underline{h} n e<$ Bedeutung zu diskutieren $>$ \\
\hline (8) & XXXV 5 izesces & hny $<$ Bedeutung zu diskutieren $>$ \\
\hline
\end{tabular}

Ferner sind noch Belege dieser Schreibung in pBM 10507 und Anchscheschonqi zu vergleichen:

\begin{tabular}{|c|c|c|}
\hline ヘУルG.S.世 & $\underline{h n} n$ ḩ3.t „, amicable ${ }^{630}$ & pBM 10507 II 11 \\
\hline 2, & $\begin{array}{l}t \underline{h} n \text {,show friendliness" } \\
\text { (oder ähnlich) }\end{array}$ & pBM 10507 II 11 \\
\hline $1,0,0$ und $<, .52$ & $\underline{h} n$ „sich nähern, Nachbar ${ }^{632}$ & $\begin{array}{l}\text { Anchscheschonqi XI } 3, \text { XX } 16=(5) \\
\text { oben }\end{array}$ \\
\hline 5,0 & $\begin{array}{l}\underline{h} n \text {,Dummkopf, } \\
\text { Taugenichts }\end{array}$ & $\begin{array}{l}\text { Anchscheschonqi XXIII 12, } \\
\text { XXVII } 3=(3) \text { oben }\end{array}$ \\
\hline
\end{tabular}

${ }^{25}$ Thissen, in: TUAT III 2, 300.

${ }^{26}$ Wb III 367, 11; 384, 2.

${ }^{27}$ Thissen, in: TUAT III 2, 293, unter Verweis auf Emendierung bei Volten, Demotisches Weisheitsbuch $56 \mathrm{f}$.

${ }^{28}$ Außerdem pInsinger III 7, IV 11, 21, IX 6, XII 12, 13, XIII 3, XXI 12, 19, 20, 22, XXII 4, XXIII 16, XXVI 4, 9, 13, XXIX 16, XXXII 24, XXXIV 12. Variante hny: XIV 23.
${ }^{29}$ Ferner pInsinger X 12, XII 6, XIII 16, 17, XVI 6, 8, XVII 21, XX 21, XXVIII 3.

${ }^{30}$ M. Smith, The Mortuary Texts of Papyrus BM 10507. Catalogue of Demotic Papyri in the British Museum 3 (London 1987) 37.

${ }^{31}$ Smith, Mortuary Texts 68.

${ }^{32}$ Thissen, Die Lehre des Anchscheschonqi (P. BM 10508) (Bonn 1984) 107.

${ }^{33}$ Thissen, Die Lehre des Anchscheschonqi 107. 
Es sind also zwei Wurzeln voneinander zu scheiden: Einmal eine Wurzel mit negativer Bedeutung (Schreibungen (1), (3) und (4)), die entsprechend eine Determinierung mit sterbendem Krieger erhält und sich mit Volten von

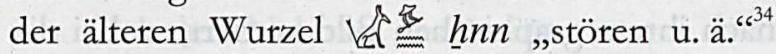
herleitet, wie die Kopenhagener Parallelen mit

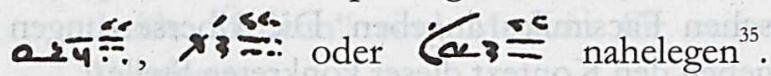
Zum anderen eine Wurzel, die mit laufenden Beinchen oder Mann mit Hand am Mund determiniert (Schreibungen (5), (6), (9) und (10)) sich auf älteres $\wedge$ 재 $h n$,herantreten ${ }^{636}$ zurückführen läßt. Schreibung (2) ist wohl aufgrund des Kontextes sicher als „Nähe“ zu übersetzen, ist aber abusiv wie „Dummheit" determiniert worden. Die Zeichen $\boldsymbol{\omega}$ und $\boldsymbol{\sigma} \boldsymbol{\epsilon}$ gelten dabei als Varianten, wobei Ersteres eine Ligatur der beiden Häkchen von Letzterem sei ${ }^{37}$, bzw. $\boldsymbol{\omega}$ nach Spiegelberg schriftgeschichtlich von $\mathbb{\pi K}$ abstamme $^{38}$. Neben $h n-$ ich möchte mich nicht auf eine hieroglyphische Entsprechung oder Wurzel festlegen, kann allerdings $\sqrt{\mathrm{K}}$ weniger nachvollziehen $^{39}$ als die Annahme einer sich verselbständigenden Ligatur von $\mathbf{5} \mathrm{zu} \boldsymbol{\omega}-$ hat das Zeichen $\boldsymbol{\omega}$ in demotischen Texten aus dem Achmim des 1. Jahrhunderts v. Chr. verschiedene hieroglyphische Wurzeln und damit auch Lautwerte:

\begin{tabular}{|c|c|c|c|c|}
\hline $\mathbb{B}$ in & 幽 & $b^{c} y(?)$ & $\begin{array}{l}\text { „verachten (?), } \\
\text { wüten (?) }\end{array}$ & Anchscheschonqi XII 11 \\
\hline \multirow[t]{5}{*}{ nasifoet } & Tqune & bny.t & „Dattelpalme ${ }^{\text {c61 }}$ & Anchscheschonqi XI 18 \\
\hline & & bne & „Schwalbe“ & $\begin{array}{l}\text { pInsinger VI } 7 \\
\text { (vgl. pCarlsberg II 3, 6: es los } 1 \text { ) }\end{array}$ \\
\hline & $\therefore S^{2}$ & $b t w$ & „Verderben“ & $\begin{array}{l}\text { pInsinger VI } 7 \text { und öfter (ähnlich bty.t } \\
\text { „Abscheu“ - pInsinger XI } 10 \text { und öfter) }\end{array}$ \\
\hline & bis & $s b q$ & „klein“ & $\begin{array}{l}\text { pInsinger VI } 7 \text { und öfter, pSpiegelberg } \\
\text { XIII } 10\end{array}$ \\
\hline & $25 y$ & $s b t$ & $\begin{array}{l}\text { „Mauer }{ }^{c c} \text { und } \\
\text { „Rüstung, Vorbe- } \\
\text { reitung }{ }^{c 42}\end{array}$ & $\begin{array}{l}\text { pSpiegelberg XII 13, R X 12, XIV 1, 4, 9, } \\
\text { XVIII } 9\end{array}$ \\
\hline$\Longrightarrow$ in & $=\underline{\underline{w}}$ & swnw & „Arzt ${ }^{643}$ & Anchscheschonqi passim. \\
\hline in & b举 & s3 & „Sohn“444 & $\begin{array}{l}\text { Anchscheschonqi XV 9, pBM } 10507 \\
\text { VIII 9, IX 10, pHarkness I 1, II 31, IV } 32\end{array}$ \\
\hline
\end{tabular}

$\mathrm{Zu} \boldsymbol{\omega}$ als $h n$ sind noch neben den oben angeführten Wörtern zu erwähnen:

\begin{tabular}{|c|c|c|c|}
\hline 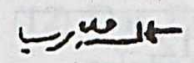 & $b \underline{h} n$ & „Turm (?), ob: prüfen, erproben? ${ }^{45}$ & Anchscheschonqi III 17, 19 \\
\hline TENone & $\underline{h} n y \cdot t$ & „Haut" & Anchscheschonqi XXI 5 \\
\hline
\end{tabular}

${ }^{34}$ Wb III 383, 3-9.

${ }^{35}$ Volten, Das demotische Weisheitsbuch 95-97.

${ }^{36}$ Wb III $373,9-18$.

${ }^{37}$ Erichsen, Glossar 382, 385.

${ }^{38}$ W. Spiegelberg, ,Beiträge zur Erklärung des Pap. Insinger", in: OLZ 31 (1928) 1036.

Siehe Stadler, SAK 29, $340 \mathrm{f}$.

${ }^{40}$ Thissen, Die Lehre des Anchscheschonqi 67.

${ }^{41}$ Thissen, Die Lehre des Anchscheschonqi 67.
${ }^{42}$ W. Spiegelberg, Der Sagenkreis des Königs Petubastis nach dem Straßburger demotischen Papyrus sowie den Wiener und Pariser Bruchstücken. Demotische Studien 3 (Leipzig 1910) 53*.

${ }^{43}$ Thissen, Die Lehre des Anchscheschonqi 110.

${ }^{44}$ Ausführliche Diskussion der Lesung bei: Smith, Mortuary Texts 67. Thissen, Die Lehre des Anchscheschonqi 111 , las $s b k$, was aber von Smith widerlegt wurde.

${ }^{45}$ Thissen, Die Lehre des Anchscheschonqi 68. 
Die Schreibungen (7) und (8) der Liste mit hn-Wörtern lassen sich nach ihren Determinativen $\boldsymbol{k}$ und $\mathbf{i}$ nicht ohne weiteres einer der beiden Gruppen zuweisen. Bei derart ähnlichen Wurzeln stellen die Determinative neben dem Kontext jedoch ein ausgesprochen wichtiges Kriterium zur Übersetzung dar. Wenn nun der Kontext wie bei pInsinger XVIII 10 (und auch XXXV 5 - bei Quack nur en passant erwähnt) selbst nicht klar ist oder zumindest einer neuen Deutung unterzogen werden soll, so bleiben die Determinative als alleinige Anhaltspunkte übrig. Sicherlich ist eine gewisse Ähnlichkeit zu den Determinativen bei $h n$,rudern. Ruderer. Auch: das Rudern ${ }^{646}$ nicht zu bestreiten. Wird diese Ähnlichkeit zu 2 trotz des zusätzlichen schrägen Striches im pInsinger akzeptiert, kann auch die Ähnlichkeit zum Determinativ von etwa ' $n$ „schön ist -, schön“, eben das äußerlich gleiche 2, nicht ohne weiteres abgelehnt werden, das bei ${ }^{\prime} n$ als transkribiert wird. Die im pInsinger deutlichen zusätzlichen Striche zu ignorieren, führt also in eine Beliebigkeit, bei der alles möglich wird.

Wenn nun die Striche aber ernst genommen werden, grenzen sich die Möglichkeiten sehr stark ein. Als Ausgangspunkt nehme ich die Schreibung inswe in pInsinger XXXV $5{ }^{47}$, deren Strich offenbar durch eine leichte Beschädigung in zwei Teile zerfällt, tatsächlich aber entspricht das Determinativ 12 in Normalschreibung. Dieses Zeichen begegnet in Schreibungen von iry "Gefährte“ als vor allem ptolemäische und dann römische Variante von

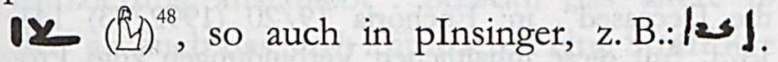
Wird nun eine Determinativübertragung angenommen und unter Berücksichtigung der Be-

${ }^{46}$ Erichsen, Glossar 383.

47 Von Quack, Enchoria 25, als Untermauerung seiner These herangezogen.

${ }^{48}$ Erichsen, Glossar 38. Vgl. P. W. Pestman/ S. P. Vleeming, Les papyrus démotiques de Tsenhor (P. Tsenhor). Les archives privées d'une femme égyptienne du temps de Darius Ier I (Leuven 1994) 137. O. El-Aguizy, A Palaeographical Study of Demotic Papyri in the Cairo Museum from the Reign of King deutung des Herkunftswortes frowe als

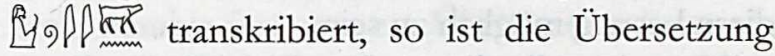
„Freund“ ein am Sinn der beiden Sätze zu überprüfender Ansatz. Unter diesen Umständen erscheint dann aber eine hieroglyphische Transkription mit $\mathrm{H}_{\text {of }}$ 焦 aus ägyptischer Sicht problematisch, wenn es auch - wie oben vermutet - aus einer Ligatur von $\delta \mathbb{C}$ (hieroglyphisch $\mathrm{zu} \omega$ entstanden und $\mathrm{s}$ of schriftgeschichtlich deswegen unter Umständen korrekter sein mag. Problematisch ist dies aber, weil so in Hieroglyphen eher der „Störenfried“ geschrieben werden würde, wenngleich im Demotischen zwischen den beiden $h n$-Zeichen für die Wurzeln „stören“ und „nähern" offensichtlich nicht mehr unterschieden wird. Die Determinativübertragung von $\mathbf{0}$ bei Wörtern des Bedeutungsfeldes „Freund, Gefährte“ wird durch römische Schreibungen für hhbr „Genosse, Freund" mit der Gruppe $\mid \boldsymbol{\Omega}\rfloor$, die für sich sonst iry „Gefährte“ gelesen wird, als Determinativ wahrscheinlich gemacht ${ }^{49}$. Die Existenz des Wortes $\underline{h n}$ „Freund“ im Demotischen ist von M. Smith bewiesen worden ${ }^{50}$. Dort bespricht er die anderen ihm bekannten Belege des Wortes, unter denen besonders die beiden des pKrall von Interesse sind (pKrall IX 32 und XIV 3: weil sie im Gegensatz zu den anderen mit Mann mit Hand am Mund oder laufenden Beinchen determinierten Schreibungen ebenfalls mit Varianten des Determinatives 12 (M) abschließen ${ }^{51}$, wobei außerdem die häufige Parallelität des Wortes $h n$ zu iry „Gefährte" im pKrall bemerkenswert ist und die Sicht der Determinativübertragung unterstützt ${ }^{52}$.

Taharka to the End of the Ptolemaic Period (684-30 B.C.). MIFAO 113 (Le Caire 1998) $296 \mathrm{f}$.

Erichsen, Glossar 354.

50 Smith, Mortuary Texts 103.

${ }^{51}$ Die Determinierung läßt sich freilich nicht ganz sicher hieroglyphisch erklären, wie das in der römischen Zeit ohnehin schwieriger wird. Es könnte sich auch um 獣, handeln.

${ }^{52}$ Erstmals E. Bresciani, Der Kampf um den Panzer des Inaros (Papyrus Krall). MPER 8 (Wien 1964) 117. Gefolgt von: Smith, Mortuary Texts 103. Vgl. auch F. Hoffmann, Der Kampf um den Panzer des Inaros. Studien zum P. Krall und seiner Stellung 
Paläographisch wie lexikographisch scheint also diese Lesung möglich zu sein.

Nun ist allerdings die Richtigkeit dieser Deutung für den pInsinger am Text zu überprüfen, indem festgestellt werden muß, ob die Stellen XVIII 10 und XXXV 5 tatsächlich mit der Übersetzung „Freund“ besseren Sinn ergeben. Die beiden Sätze hießen somit wie folgt:

XVIII 10: hne iw $b w$-ir $=f n^{c} p 3$ nt mwhmh $n$ n3y=f iwf.w

„Ein Freund, der nicht gnädig ist, ist der, der sein Fleisch *malträtiert."

XXXV 5: $ヶ s=y n p 3 n t r h t p=f-n=y$ ir $=f-n=y \underline{h n y} i w$ $m n[\ldots]$

„Ich rufe zu Gott, möge er mir gnädig sein, möge er mir ein Freund $\operatorname{sein}^{53}$, in dem es nicht gibt $[\ldots]^{\text {“" }}$

Den beiden Stellen zufolge ist also der Freund a) der $w r$ iy $h<. w>$ "Oberste der Ach-Geister ${ }^{654}$, was aus pInsinger XVIII 8 folgt, auf den sich XVIII 10 bezieht, b) allgemein göttlicher Natur. Als Oberster der Ach-Geister kommen in der Unterwelt zwei Götter in Frage, einmal natürlich Osiris, zum anderen aber auch Anubis, auf dessen Königsqualitäten J. Quaegebeur hingewiesen hat $^{55}$. Der Oberste der Ach-Geister kann in der Unterwelt ein Verstorbener in Annäherung an Anubis werden, wie aus pBM 10507 und der Parallele pHarkness deutlich wird: $i r=k$ mre $n n 3$ in-mwt.w wr $n n$ 3 ihy.w „Du wirst als Vorsteher der Verstorbenen und Oberster der Ach-Geister handeln" (pBM 10507 X 3) bzw. $t w=t$ (n) $m r-i h$ $n 3$ ihy.w wr n3 in-mwt.w „Du bist ein Rindervorsteher der Ach-Geister und Oberster der Ver-

innerhalb des Inaros-Petubastis-Zyklus. MPER 26 (Wien 1996) 226 mit Anm. 1177.

${ }^{53}$ Als freiere Übersetzung von entweder wörtlich „möge er mir einen Freund machen", oder mit einer kleinen Ergänzung: $i r=f-n=y(n)$ hny ,möge er für mich (als) ein Freund handeln".

„Oberdämon" ist im Deutschen etwas problematisch, weil „Dämon“ negativ konnotiert ist. Daher bleibe ich bei dem etwas holperigen „Obersten der AchGeister".

${ }_{55}$ J. Quaegebeur, „Lettres de Thot et Décrets pour Osiris", in: J. H. Kamstra/H. Milde/K. Wagtendonk (Hgg.), Funerary Symbols and Religion. Fs M. S. H. G. Heerma van Voss (Kampen 1988) 105126. storbenen." (pHarkness II 36) ${ }^{56}$. Dabei scheint die Konstruktion proklitisches Pronomen $+m$ (bzw. $n$ ) of predication + Nomen/Nomina auf eine gewisse Distanz zwischen den zwei durch $m / n$ verbundenen Elementen hinzuweisen, d. h. "Vorsteher" bzw. „Oberster" sind vielmehr erworbene, verliehene Attribute als unveräußerliche Merkmale des/der Verstorbenen, was durch die in pHarkness unmittelbar folgende Konstruktion unabhängiges Pronomen + Nomen ausgedrückt werden würde. Die Stelle des pHarkness ist aber auch aus einem anderen Grund bedeutsam, denn hier steht einmal ein Titel des Anubis ( $m r-i h$,Rindervorsteher $\left.{ }^{6}\right)^{57}$ verbunden mit den Ach-Geistern und $w r n 3$ in$m w t . w$ „Oberster der Verstorbenen“ nebeneinander, wobei $w r n 3$ in-mwt.w nahe an den $w r$ $i y h<. w>$ „Obersten der Ach-Geister" des pInsinger herankommt.

Von den beiden Alternativen Osiris und Anubis fügt sich am besten Anubis ein, weil in Kolumne XVIII erstens deutlich auf die Einbalsamierungsprozedur hingewiesen wird, und zweitens Anubis wiederum in pBM 10507 VIII 5 als der Freund des Verstorbenen herannaht: $i w-n=k$ inpw $\underline{t}=f-t=k n \underline{h} n$,Anubis wird zu dir kommen, so daß er dich als Freund nehmen möge."

Damit kommen bei der Übersetzung hnne/hny „Freund“ zwei Eigenschaften zusammen, die

${ }^{56}$ Vgl. den Kommentar von Smith, Mortuary Texts 115, dazu. Zur Psychopompos-Funktion des Anubis, die darin zum Ausdruck kommt, vgl. M. Smith, „A Demotic Formula of Intercession for the Deceased", in: Enchoria 19/20 (1992/93) 141, 152f. Auf diese inhaltlichen Vërbindungen zum Problem des pInsinger hat mich Dr. M. Smith aufmerksam gemacht. Ferner stammt die von der publizierten Version abweichende Lesung $m r-i h$ "Rindervorsteher" statt mry für pHarkness II 36 ebenfalls von ihm.

57 Anubis wird in pHarkness I 9 mit dem $m r$-ih identifiziert $\left(m \varsigma^{c}=t\right.$ irm inpw ir=t $h b r n p 3 \quad m r$-ih , $\mathrm{Du}$ wirst mit Anubis wandeln. Du wirst ein Gefährte des Rindervorstehers sein.") (Hinweis Dr. M. Smith). Schon Erichsen, Demotisches Glossar 166. S. außerdem zu Anubis als Rindervorsteher: J. Quaegebeur, „Anubis, fils d'Osiris, le vacher", in: StudAeg 3 (1977) 119-130. Dort zahlreiche weitere Belege auf S. 123. Ein jüngst publizierter Beleg: J. Osing, Hieratische Papyri aus Tebtunis I. The Carlsberg Papyri 2 (Copenhagen 1998) 172f., 177 Anm. p. 
auf Anubis hinweisen und erhellendes Licht auf die Deutung von pInsinger XVIII 5-12 werfen. Demnach wird dort vor den Qualen gewarnt, die einem Geizigen nach dessen Tod widerfahren können, indem die sonst durch die Balsamierung erhofften Wohltaten sich zu schmerzhaften Prozeduren verkehren. Sogar der sonst als Freund anzusehende balsamierende Anubis ist erbarmungslos.

Doch lassen sich die inhaltlichen Auswirkungen noch weiter verfolgen, weil Anubis seit den Pyramidentexten bis in die ptolemäischrömische Zeit hinein als Richter in der ägyptischen Totenreligion auftrat ${ }^{58}$. Dabei erscheint er sowohl als einer, der einem Kollegium von sieben. Ach-Geistern vorsteht ${ }^{59}$, unter denen die Horussöhne an erster Stelle stehen, die für den Schutz des Osiris-Leichnames verantwortlich sind und die mit den Schüzgeistern in den Stundenwachen identifiziert werden können, als auch in richterlicher Funktion. Im Gegensatz zu der älteren Auffassung, daß der Beiname ip ib.w „der die Herzen bewertet" nicht in diese Richtung weist, kann Willems sehr wohl die richterliche Bedeutung dieses Beinamens nachweisen, weil ip ib.w von der in ptolemäisch-römischer Zeit beliebten Charakterisierung $i p$-ib „verständig“ ${ }^{\text {" }} \mathrm{zu}$ trennen sei. In diesem Lichte wird Quacks Vorschlag von „untersuchen, examinieren" für shyh in pInsinger XVIII 11 untermauert $^{60}$, weil es m. E. eine Anspielung auf dieses ip ib.w „der die Herzen bewertet“ und damit auf die Tätigkeit des Anubis beim Wägen des Herzens ist, die sich - wie auch im pInsinger - unmittelbar an die von Anubis durchgeführte $\mathrm{Mu}$ mifizierung anschließt $t^{61}$. Sowohl das ältere ip $i b . w$ als auch das shyh implizieren eine Straffolge der Untersuchung, um den Schutz des Osiris zu

${ }^{58} \mathrm{H}$. Willems, "Anubis as a Judge“, in: W. Clarysse/A. Schoors/H. Willems (Hg.), Egyptian Religion. The Last Thousand Years. Studies Dedicated to the Memory of Jan Quaegebeur I. OLA 84 (Leuven 1998) 719-743.

${ }^{59}$ Vgl. dazu J.-Cl. Goyon, „Momifications et recomposition du corps divin: Anubis et les canopes", in: Fs Heerma van Voss 34-41.

${ }_{60}$ Quack, Enchoria 25, 30.

${ }^{61} \mathrm{Zu}$ Herzwägung und Mumifizierung im Zusammenhang mit Anubis bes. Willems, Gs Quaegebeur I $730-736$. gewährleisten. In seiner richterlichen Funktion stellt Anubis fest, wer ein Übeltäter und wer ein Rechtschaffener ist, um nur letzteren zu Osiris zu lassen, ersteren aber zu bestrafen. Im Sargtextspruch 781 bewertet Anubis die Herzen ( $i p=f-n=k i b . w=s n$ ) und wird gleich anschließend als hry s3w.w „Oberster der Wächter“ angesprochen, der für den Toten die gegen ihn Handelnden zerstückeln soll ( $\varsigma^{r}=f$ ir $r=k$ hry $\left.s 3 w \cdot w\right)^{62}$.

Um nochmals die einzelnen Argumentationsfäden aufzunehmen: Paläographisch und lexikographisch kann /20 als hne „Freund“ genommen werden. Dieser Freund ist Anubis, der als Psychopompos dem Toten ein Freund ist und ihn bei der Hand nehmend geleitet. Der zuvor genannte $w r$ iyh $h<w\rangle$ „Oberste der AchGeister" ist ebenfalls als Anubis zu verstehen, der spätestens seit den Sargtexten Schutzgeistern vorsteht, als hry s3w.w „Oberster der Wächter“ etwa in den Sargtexten oder $m r$-ih oder mre $n n 3$ ihy.w „Rindervorsteher" oder „Vorsteher der Ach-Geister" und wr in-mwt.w "Oberster der Verstorbenen" in demotischen Funerärtexten. Auch die im pInsinger dem hne folgende Charakterisierung des Gottes als einer, der strafrechtlich examiniert (shyh), läßt sich mit der richterlichen Funktion des Anubis vergleichen, der sonst ip ib.w „der die Herzen bewertet" ist. Genauso, wie in den älteren von Willems zusammengestellten Quellen diese von Anubis durchgeführte Untersuchung im Zusammenhang mit der Mumifizierung zu sehen ist, wird auch im pInsinger die Einbalsamierung thematisiert, um dann nach einem Examen zur Strafexekution des Übeltäters zu kommen. Doch dann ist zu fragen, ob die Vermutung von Willems, daß der in der Saitenzeit wiederentdeckte Begriff ip ib.w anders verstanden und reinterpretiert wurde, grundsätzlich richtig ist. Willems deutet die Beleglage nämlich dahingehend, daß in den Pyramiden- und Sargtexten mit ip ib.w gemeint sei, die Herzen der sethischen Feinde des Osiris zu kontrollieren, um sie physisch unschädlich zu machen. Ab der Saitenzeit sei aber der Begriff ip ib.w in Zusammenhang mit dem Wägen des Herzens gesehen worden. Im

\footnotetext{
${ }^{62}$ CT VI $412 \mathrm{~b}-\mathrm{c}$.
} 
pInsinger hingegen scheint mir die Kontrolle im Sinne der älteren ägyptischen Totenliteratur noch nachzuweisen zu sein, denn der nach dem pInsinger von Anubis gequälte Übeltäter $(s 3 b 3$ „Frevler, Feind“) dürfte doch wohl als ein Sethanhänger zu verstehen sein, der von Osiris ferngehalten werden soll.

In dieser Interpretation werden nicht nur paläographische Probleme ausgeräumt, sondern auch der Text inhaltlich verständlicher und sein Zusammenhang zur älteren und zeitgenössischen religiösen Literatur deutlich. Der Balsamierer ( $w r$ iry), der zwischenzeitlich statt des $w r$ $i y h<. w>$ „Oberster der Ach-Geister" im pInsinger gesehen wurde, ist nun zwar wieder aufgrund der Paläographie zum Obersten der AchGeister geworden, doch dieser ist als der balsamierende Anubis identifiziert worden. In der im vorliegenden Aufsatz behandelten Stelle stehen somit die Unheilsdämonin $t 3$ wr3.t und der Oberste der Ach-Geister einander gegenüber. Sogar er, der sonst der Freund des Verstorbenen ist, steht dem verstorbenen Geizigen feindlich gegenüber. Es tritt uns außerdem der Humor des Autors entgegen, der in einer ironischen Bemerkung die totenreligiösen Praktiken mit gewisser Skepsis betrachtet. Da aber der Verstorbene Pa-Heb, Sohn des Dje-her-pa-a'an, die Lehre des pInsinger als Grabbeigabe bei sich hatte, belegte er, daß er eben nicht geizig war, und hatte deshalb keinen Grund - so dürfen wir hoffen -, die Qualen der Balsamierung zu fürchten.

\section{SUMMARY}

Attention is re-drawn to the colophon of pInsinger through which it becomes apparent that this papyrus was part of a burial equipment. The implications for the general interpretation of the instruction are briefly discussed. The second part deals once again with the problem of pInsinger XVIII 5-12 and its understanding. The translation hne 'boat-trip' in XVIII 10 is being rejected, and the rendering 'friend' is being proposed instead. This ironical allusion to Anubis turns pInsinger XVIII 5-12 into a treatment of a judgement in the hereafter which Anubis presides. 